\title{
An evaluation of two commonly used tests of unfamiliar face recognition
}

\author{
Bradley C. Duchaine ${ }^{\mathrm{a}, *}$, Andrea Weidenfeld ${ }^{\mathrm{b}}$ \\ a Department of Psychology, Vision Sciences Laboratory, Harvard University, 33 Kirkland Street, William James Hall, \\ 7th Floor, Cambridge, MA 02138, USA \\ ${ }^{\mathrm{b}}$ Institut für Psychologie der Universität Potsdam, Potsdam, Germany
}

Received 19 March 2001; received in revised form 25 September 2002; accepted 25 September 2002

\begin{abstract}
The Warrington Recognition Memory for Faces (RMF) and the Benton Facial Recognition Test (BFRT) are commercially available tests that are commonly used by clinicians and cognitive neuropsychologists to evaluate unfamiliar face recognition. Yet, it is not clear that a normal score on either instrument demonstrates normal unfamiliar face recognition. Because the RMFs stimuli contain abundant non-internal facial feature information, subjects may be able to score in the normal range without using internal facial features. On the BFRT, subjects commonly rely on feature matching strategies using the hairline and eyebrows rather than recognizing the facial configuration. To test whether these routes to recognition can support normal performance, normal subjects were tested with versions of the RMF and the BFRT in which the faces had been painted over in a way that prevented the operation of some of the procedures normally involved with face recognition. Even though these modifications removed all of the internal feature information in the RMF, many subjects scored in the normal range, and despite precluding the use of configural processing in the BFRT, many of the scores were in the normal range. As a result, it is apparent that normal scores on these tests do not demonstrate normal unfamiliar face recognition and so clinicians should be cautious in interpreting scores in the normal range. Finally, these results place in question models supported by dissociations involving normal performance on these tests.
\end{abstract}

(C) 2002 Elsevier Science Ltd. All rights reserved.

Keywords: Prosopagnosia; Neuropsychological tests; Warrington; Benton; Object recognition

\section{Introduction}

The Warrington Recognition Memory for Faces (RMF) [51] and the Benton Facial Recognition Test (BFRT) [8] are commercially available tests that are widely used by both clinicians and cognitive neuropsychologists to assess face recognition abilities. However, there is reason to question whether normal scores on these tests actually demonstrate normal unfamiliar face recognition. The photos in the RMF include many non-internal facial features, such as clothing and hair, by which they can be recognized. This raises the possibility that participants-in particular individuals with face recognition impairments-might be able to do well on the RMF by using a strategy that does not require the integrity of normal face recognition processes. The BFRT has the same problem, but for different reasons. Because it asks participants to match faces that are presented simultaneously, participants could answer correctly by matching individual features in a piecemeal fashion [41,42]. This would

\footnotetext{
* Corresponding author. Tel.: +1-617-495-3884; fax: +1-617-495-3764.

E-mail address: brad@wjh.harvard.edu (B.C. Duchaine).
}

not, however, show that face recognition processes are intact, because it appears that face recognition is performed by both parts-based procedures that represent facial features and configural processing procedures that represent the spatial relations of the parts of the face [14,25,26,37,40,44]. Moreover and possibly more importantly, target faces and test items in the BFRT are presented simultaneously so participants are not required to rely on a memory trace.

As a result of these concerns about the RMF and BFRT, we conducted tests exploring these possibilities with modified versions. In our version of the RMF, parts-based and configural procedures could not be used on internal feature information, and our version of the BFRT precluded the use of configural procedures. If subjects can achieve a normal score nevertheless, then it will be clear that normal scores do not necessarily demonstrate normal face recognition abilities.

\subsection{Recognition Memory for Faces}

The RMF was designed as a test of non-verbal memory, but it is often used as a test a particular type of non-verbal 
memory - face memory. Subjects in the RMF are presented with black and white photos of 50 unfamiliar men at a rate of one item every $3 \mathrm{~s}$, and they are asked to respond 'yes' or 'no' according to whether they find the face pleasant or not pleasant. Immediately following presentation of the target photos, memory for the photos is tested with a forced choice between a target photo identical to that presented earlier and a distracter photo. This means that stimulus recognition, not true face recognition, is possible [29]. The number of correct choices determines the score of the subject, so the maximum score is 50. Means range between 42.4 and 44.3 depending on the age group with standard deviations of approximately 3.5 .

Even though the RMF is used to test face recognition, the photos contain information that is not internal facial feature information and could be used to discriminate between target and distracter photos. When attempting to test face recognition, the distinction between information handled by face recognition procedures and other information is clearly crucial. Although a number of investigations of face recognition have proposed that both the internal features (eyes, nose, mouth regions) and the external features (hair, ears) are recognized by face recognition procedures [21,22,54], neuropsychological evidence paints a different picture. Humans appear to use special procedures for face recognition $[11,24,31,37,40]$, and the input from the face that these procedures typically operate on defines what counts as facial information. CK, an object agnosic with normal face recognition, has shown in a number of experiments that his recognition of famous faces from the internal features is normal, but his recognition of famous faces from external features is severely impaired [39,40]. Conversely, prosopagnosics often rely on the external features, in particular the hair, for person recognition $[1,5,13,20,23,32,36,41,46]$, although their proficiency with these features has not been formally demonstrated. Thus, although the external features can obviously contribute to person recognition and appear to be more important than internal features when learning to recognize a particular person $[21,22,54]$, they do not appear to constitute part of the face for these specialized procedures.

As a result of these considerations, it appears that all information in the RMF other than the internal facial features would not be handled by specialized procedures. The photos consist of shots that display each model's hair, face, and approximately one-third of their upper body (see Fig. 1). The hair, clothing, head postures, and body positions vary greatly between the models. In addition, some photos contain missing corners, low brightness levels, or developmental imperfections.

Recent experiments with a developmental prosopagnosic vividly illustrate the problem caused by the presence of the non-internal feature information [42]. EP's performance was clearly impaired on tests of familiar and unfamiliar face recognition. However, EP was able to achieve a normal score on the RMF, and he reported using the non-internal feature information. In order to investigate his claim, EP

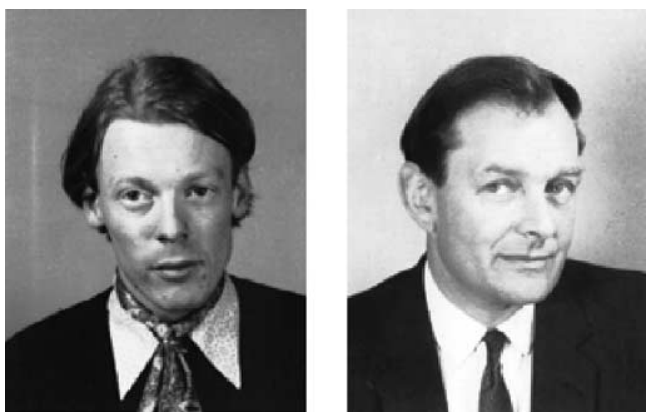

Fig. 1. Example of a forced-choice pair in the RMF. Copyright Elizabeth K. Warrington, 1984. Reproduced by permission of NFER-NELSON, Windsor, UK.

was presented with the RMF without the information around the face. Not surprisingly, the mean for the control subjects was only two points lower in the modified version, because they were relying primarily on the internal feature information. In contrast, EP scored 31/50 on the modified test, and so it appears that the non-internal feature information does provide a route to normal performance.

\subsection{Benton Facial Recognition Test}

Two versions of the BFRT [8] can be used depending upon time considerations: the Short Form has 13 items with 27 possible points while the Long Form has 22 items with 54 possible points. On each item, subjects are presented with a target photo, and they are asked to choose the target individual from six faces presented simultaneously with the target photo (see Fig. 2). There are three parts to the BFRT: (1) matching a frontal view of the target with an
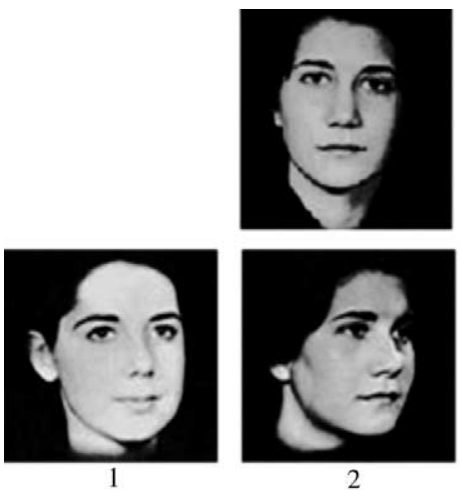

2

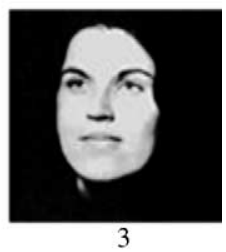

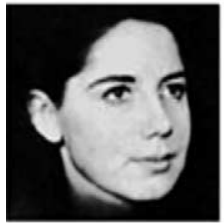

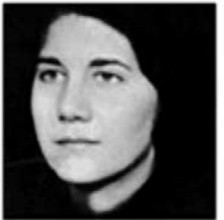

5

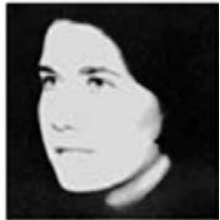

6
Fig. 2. Example of a BFRT item. Subjects are to select three photos that depict the individual pictured above (2, 5, and 6). 
identical photograph, (2) matching a frontal view of the target individual with three photos of the target taken from different angles, and (3) matching a frontal view of the target individual with three photos of the target taken under different lighting conditions. No time limits are placed on the BFRT, and scores are classified as normal when 41 or above.

The black and white photos used in the BFRT consist of unfamiliar male and female faces with their hair and clothing shaded out so that subjects must rely on the face. On many of the items, the features that best differentiate the photographed individuals are the eyebrows and hairlines: normal subjects and prosopagnosic subjects to whom we have administered the test, as well as prosopagnosics in [20,41,42], have reported that their performance relied heavily on matching the eyebrows and hairlines rather than the internal facial features.

\subsection{Assessing the RMF and the BFRT}

Because of the possibility that normal scores on the RMF and the BFRT do not reflect normal face recognition abilities, we have designed experiments that will determine how well participants can perform on these tasks without using all of the processes involved with normal face recognition. For the RMF, we covered the faces in the test phase so that participants were forced to rely on only the non-internal feature information available in the photos (see Fig. 3). For the BFRT, we covered all of the face except for the eyebrows and the hairline, so that participants could not use the facial configurations for matching (see Fig. 4). We will refer to these modified versions of the task as the mRMF and the mBFRT.

Because we deleted internal feature information, this allows us to test normal subjects under conditions similar to those experienced by individuals with normal parts-based processing but without facial configural processing. However, we deleted internal features that could have been used by parts-based processing so our versions provide an especially demanding test of the hypothesis that these tests can be passed using feature-based procedures. If some
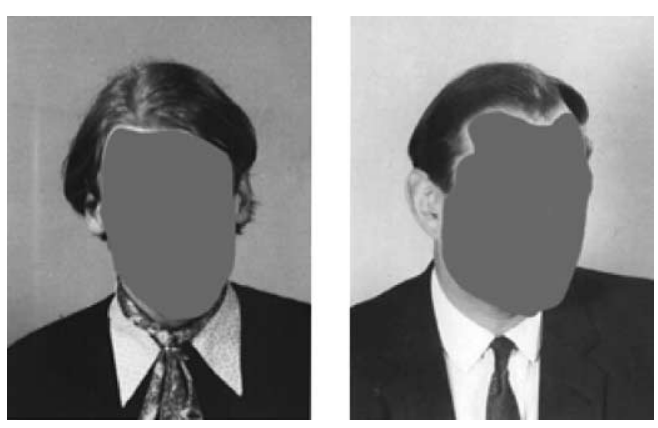

Fig. 3. The forced-choice pair from Fig. 1 in its modified form. Copyright Elizabeth K. Warrington, 1984. Reproduced by permission of NFER-NELSON, Windsor, UK.

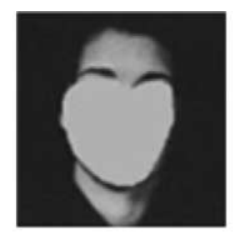

7
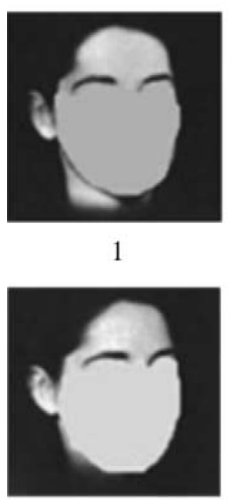
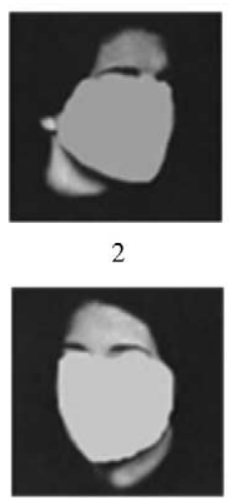
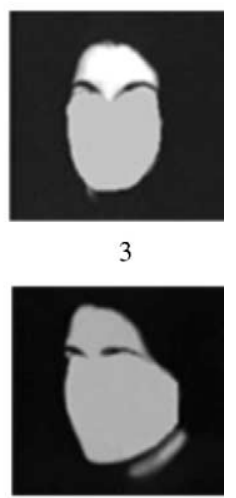

6
Fig. 4. The BFRT item presented in Fig. 2 in its modified form.

participants are able to perform in the normal range on either test, it will indicate that the original test does not require normal face recognition abilities. However, if participants have scores well out of the normal range, it will indicate that the original tests do, in fact, require intact face recognition.

\section{Experiment 1a: modified Recognition Memory for Faces (mRMF)}

\subsection{Method}

\subsubsection{Participants}

The participants were 26 undergraduate students, 17 women and 9 men, who participated to satisfy a requirement for their introductory psychology class at UC-Santa Barbara.

\subsubsection{Materials}

The stimuli were digitized versions of the stimuli from the Warrington Recognition Memory for Faces test. The 50 two-choice test stimuli were scanned into a computer so that the faces could be painted over with a shade of gray. The gray extended from each model's hairline to his chin vertically and horizontally from ear to ear or from sideburn to sideburn, if sideburns were present (see Fig. 3). We then copied the target stimulus from each of the forced-choice pairs for use in the study phase of the experiment. This insured that each target stimulus shown during the study phase was identical to the target stimulus used in the test phase. Digitized stimuli were the same size as the stimuli in the RMF, and each stimulus pair in the test phase was presented side by side with the same spacing used in the normal RMF. 


\subsubsection{Procedure}

Participants were seated at computers and were presented with instructions on the screen. The instructions informed them that they would view people with their faces painted over, but pointed out that there was a large amount of non-internal feature information available to utilize for the recognition test that would follow. Participants were encouraged to use any mnemonic strategies they felt comfortable with, and it was suggested that giving the target individuals names or noting imperfections in the photos might be effective methods. ${ }^{1}$

Following the instructions, participants were presented with the 50 target stimuli for $3 \mathrm{~s}$ each. A white screen was presented for $1 \mathrm{~s}$ between each target. At the end of the study phase, participants read instructions directing them to right click on their mouse if the target was on the right and to left click if the target was on the left. If they were unsure which stimulus was the target, they were instructed to guess. As in the normal RMF, reaction time was not measured. However, we required that the participants make their decision within $10 \mathrm{~s}$. After reading these instructions, participants responded to the 50 test pairs.

\subsection{Results}

Because the participants were college age, the scores were compared to the 18-39-year-old sample provided in the RMF manual. Scores of 38 or above are classified as normal, and 17 of the 26 participants would be classified as normal on the basis of their scores on the mRMF. In other words, in the absence of internal feature information, $65 \%$ of the participants were able to achieve a score that would label them as unimpaired on unfamiliar face recognition. The average for the participants was 38.5 with a standard deviation of 4.04 .

\section{Experiment 1b: modified Recognition Memory for Faces (mRMF)}

The previous experiment showed that the non-internal feature information alone provides enough information for participants to score in the normal range. However, the procedure differed in a few respects from the standard RMF, and these differences may have inflated the scores on the mRMF. First, participants were not required to make a 'pleasant/unpleasant' decision with regard to each face. Although past experiments indicate that such a decision produces better face recognition performance than an intentional memory strategy [10,52], it may have raised scores in our test. Second, we presented stimuli in the

\footnotetext{
1 Our instructions varied in a number of respects from the regular instructions, but we did this to encourage subjects to use strategies that individuals with face recognition impairments use to compensate for their deficits.
}

study period that only had non-internal feature information; of course, the RMF study stimuli had internal facial features and non-internal feature information. Because faces are stimuli that are especially likely to capture attention $[34,49,50]$, omission of the internal feature information may have made the non-internal feature information easier to attend to and remember than it would be in the standard RMF.

In order to determine if these differences inflated the scores of the participants, we have modified our procedure with the mRMF in experiment $1 \mathrm{~b}$. Participants are required to make a 'pleasant/unpleasant decision', and the study stimuli are the normal study stimuli that have both internal features and the non-internal feature information. If participants are still able to score in the normal range, this will reinforce our previous results and eliminate the concerns discussed above.

\subsection{Method}

\subsubsection{Participants}

The participants were 23 undergraduate and graduate students, 10 males and 13 females, from Harvard University who participated for a small payment.

\subsubsection{Materials}

The test stimuli in this version of the mRMF were the same as the previous experiment. The study stimuli were created by copying the target stimulus prior to painting over the internal feature information from each test pair. This assured that the study and test versions of the target were identical other than the deletion of the internal feature information in the test stimuli.

\subsubsection{Procedure}

The procedure was identical to the previous procedure except that participants were required to make a 'pleasant/unpleasant' decision after viewing each study stimulus. Participants indicated their decision with one of two keyboard responses. As in the previous experiment, the study stimulus was presented for $3 \mathrm{~s}$. A white screen was presented immediately following each study stimulus, and it remained until participants made the 'pleasant/ unpleasant' decision after which the next study stimulus appeared.

\subsection{Results}

Scores of 38 or above are considered in the normal range, and eight of the 23 participants scored in the normal range. This proportion-35\%-was less than that seen in experiment $1 \mathrm{a}$, but the non-internal feature information still allowed more than one-third of the participants to achieve a normal score. The average score was 35.4 with a standard deviation of 4.68 . 


\section{Experiment 2: modified Benton Facial Recognition Test (mBFRT)}

\subsection{Method}

\subsubsection{Participants}

The participants were 29 undergraduate students, 10 males and 19 females, from UC-Santa Barbara who participated to satisfy a requirement for their introductory psychology course.

\subsubsection{Materials}

In order to eliminate the possibility of configural face processing, the stimuli from the BFRT were modified to leave only the eyebrows and the hairline visible. After scanning the original test items, gray masks were painted over the face of each model up to the eyebrows (see Fig. 4). The modified stimuli from each item on the BFRT were then placed on a common canvas in the same configuration as stimuli in the BFRT: the target stimulus was placed above two rows of test stimuli. As in the BFRT, numbers were placed below each test stimulus so that subjects could easily identify each stimulus.

\subsubsection{Procedure}

Participants were seated at a computer, and read the instructions for the task on the monitor. They were informed that for the first six problems there was only one correct answer and that the remaining problems had three correct answers. Participants were provided with an answer sheet on which they were to circle the stimulus numbers that they perceived to match the target stimuli. Like participants in the standard BFRT, they were allowed to proceed at their own pace.

\subsection{Results}

One point is awarded for every correctly matched test stimulus. There are six possible points on the first six items and 48 possible points for the remaining 16 items for a total of 54 points. The Short Form consists of the first 13 items, and it has a total of 27 possible points. The Long Form consists of all 22 items, and so there are 54 possible points.

Because participants responded to every item on the mBFRT, each subject produced a score for the first six items, a Short Form score, and a Long Form score. For the first six items, the target and the matching test item were identical images, and the participants' average of 5.85 showed that they had very little trouble with these items. On the Short Form, participants averaged 20.41 points with a standard deviation of 2.15. A Short Form score of 20 converts to 41 on the Long Form, and 41 is classified as a normal score. Inspection of individual scores shows that the performance of 16 of the 27 subjects on the Short Form would be categorized as normal. On the Long Form, participants averaged 40.04 points with a standard deviation of 3.35 . Scores of
39-40 are classified as borderline. Eleven of the 27 participants had scores of 41 or above, which places them in the normal range on the Long Form. Therefore, with only the hairline and eyebrows, 59\% of the participants would be classified as normal using the Short Form and $41 \%$ would be considered normal using the Long Form.

After the first six problems, test images differed either in the illumination of the faces or in the orientation of the faces. There were eight items of each type, and scores were higher for the orientation changes than for the lighting changes. The mean for the sum of the orientation change items was 18.89 with a standard deviation of 1.95 while it was 15.30 with a standard deviation for the 2.23 for the lighting changes.

\section{Discussion}

In order to assess the RMF and the BFRT, we tested participants with modified versions of each test. Test faces in the mRMF were painted over so that they provided only non-internal feature information; the stimuli in the mBFRT presented only the eyebrows and the hairline. The mRMF did not allow either parts-based procedures or configural processing to operate on internal feature information in the test phase, and the mBFRT did not permit configural processing. Despite this, many participants scored in the normal range on both tests. It bears noting that individuals with face recognition impairments are forced to rely on alternative routes to face recognition, and so can be expected to be particularly adept at using them for recognition. In contrast, the participants in this experiment presumably depend on these other cues less often. Thus, one might expect prosopagnosics without other perceptual impairments to perform better on the RMF and the BFRT than the normal participants did on the mRMF and the mBFRT. The results, which show that normal scores on these tests do not demonstrate normal unfamiliar face recognition, have two major implications. First, neither the RMF nor the BFRT, at least in their present form, should be used to diagnose unfamiliar face recognition without additional tests. Second, it is necessary to reconsider aspects of theories of normal face recognition for which performance on the RMF or the BFRT served as critical evidence.

\subsection{Recognition Memory for Faces}

The results with the mRMF clearly showed that the noninternal feature information was rich enough to support scores in the normal range. Although scores were lower in the second experiment with the mRMF, many participants were still able to score in the normal range. In addition, because our test stimuli did not have internal features, there were fewer features for feature-based procedures to operate on. Our results then raise the question of whether subjects actually do, in fact, use this information. The performance of the prosopagnosic tested by Nunn et al. [42] clearly showed that individuals sometimes do use this information. 
This report and our results suggest that normal scores achieved with the RMF may not, in fact, indicate intact face recognition abilities. However, it does not cast doubt on testing done with the RMF showing impaired performance, because individuals with impaired scores were unable to perform normally with both the internal features and the noninternal feature information present.

\subsection{Benton Facial Recognition Test}

Our results with the mBFRT showed that the presence of only the eyebrows and the hairline were sufficient to support normal performance. Subjects were able to match these features in a piecemeal fashion by comparing the simultaneously presented target photograph and test photographs. Because our stimuli did not permit the operation of configural face processing, a normal score on the BFRT does not demonstrate normal unfamiliar face recognition abilities. This conclusion is also supported by reports of prosopagnosics with impaired unfamiliar face recognition who have scored normally on the BFRT $[20,41,42]$. These individuals have typically reported that they performed the task with the same feature matching strategy that our normal participants were forced to use in the mBFRT. Of course, our results show that normal scores on this test may not reflect intact face recognition processes. However, as was the case with the RMF, impaired performance does indicate impaired face recognition and so results out of the normal range on the test are informative.

Despite the problems that we have found with the BFRT, there are changes that could be made to improve it as a test unfamiliar face perception. When prosopagnosics have achieved normal scores, the investigators have often reported that they took a long time to complete the test $[20,41,42]$. This suggests that reliance on a feature matching strategy requires more time, and so it may be possible to test configural face perception by adding time norms or by limiting the time allowed for each item. In order to probe unfamiliar face recognition rather than face perception, tests should have memory requirements, and this can be done by presenting the target and test stimuli from the BFRT in a sequential rather than a simultaneous manner.

\subsection{Implications for theories of normal face recognition}

Results from the RMF and the BFRT have served as critical pieces of evidence in theories of face recognition. In particular, normal performances on either the RMF or the BFRT coupled with impaired familiar face recognition have provided most of the cases serving as one half of the double dissociation between unfamiliar face recognition and familiar face recognition. This double dissociation led researchers to postulate separate procedures for these abilities $[6,7,11,53]$. It is most clearly articulated in the Bruce and Young model [11] of face recognition. A module housing face recognition units is used to recognize familiar faces. However, when unfamiliar face recognition is required, the model proposes that a module referred to as Directed Visual Processing is used to temporarily store and recognize these faces. So, even though familiar face recognition and unfamiliar face recognition are computationally similar problems, this model posits that they are handled by separate systems in order to account for the neuropsychological data.

Forty-two individuals have been reported who showed normal unfamiliar face recognition with impaired familiar face recognition. ${ }^{2}$ In 22 of these cases, unfamiliar face recognition was tested only with the BFRT and/or the RMF $[4,5,9,12,16,17-20,23,28,30,35,36,47,48]$. Because we have demonstrated that normal scores on these tests do not require intact unfamiliar face recognition, these cases should no longer be considered support for the dissociation. The other 20 cases used either other unfamiliar face recognition tests or other unfamiliar face recognition tests in conjunction with the RMF or the BFRT $[2,3,15,33,38,43,45]$. All of the tests of unfamiliar face recognition used in the reports had shortcomings that imperil their interpretation. The tests were either simultaneous matching tasks like the BFRT which allow feature matching or included significant non-internal feature information. In addition, response time data were never collected in the experiments supporting the dissociation, and so it is possible that abnormally long response times may have allowed impaired participants to achieve normal accuracy scores [27,55].

Therefore, in every case appearing to show evidence for normal performance on unfamiliar face recognition tasks with impaired performance on familiar face recognition tasks, the evidence is open to question. Until subjects are tested using better designed tests of unfamiliar face recognition and found to show the dissociation, there is no unequivocal support for this half of the purported double dissociation between unfamiliar and familiar face recognition exists. Theories proposing the existence of a module for each task were driven by the apparent double dissociation between the tasks, so when this dissociation is undermined it is unnecessary to propose multiple modules. In order to incorporate these considerations in a modified version of the Bruce and Young [11] framework, we suggest that the face recognition units recognize both familiar faces and unfamiliar faces.

\section{Acknowledgements}

Advice and encouragement were provided by Leda Cosmides, John Tooby, and Ken Nakayama, and we also wish to thank Elizabeth Levy, Cathy Galvez, and Crystal McMillan for their assistance in constructing and running these

\footnotetext{
${ }^{2}$ Although these articles all reported cases with normal unfamiliar face recognition and impaired familiar face recognition, the authors of these articles often did not argue that these dissociations manifested evidence for separate mechanisms for the two types of recognition.
} 
experiments. These studies were supported by the James $\mathrm{S}$. McDonnell Foundation, the NSF (Grant BNS 9157-499), a UCSB Research Across Disciplines Grant, and the NIH (Grant 1 F32 MH64246-01).

\section{References}

[1] Ariel R, Sadeh M. Congenital visual agnosia and prosopagnosia in a child: a case report. Cortex 1996;32:221-40.

[2] Assal G. Regression des troubles de la reconnaissance des physionomes et de la memoire topographique chez un malade opere d'un hematome intracerebral parieto-temporal droit. Revue Neurologique 1969;121:184-5.

[3] Barbarotto R, Capitani E, Spinnler H, Trivelli C. Slowly progressive semantic impairment with category specificity. Neurocase 1995; 1:107-19.

[4] Bauer RM. Autonomic recognition of names and faces in prosopagnosia: a neuropsychological application of the guilty knowledge test. Neuropsychologia 1984;22:457-69.

[5] Bentin S, Deouell L, Soroker N. Selective visual streaming in face recognition: evidence from developmental prosopagnosia. NeuroReport 1999;10:823-7.

[6] Benton AL. The neuropsychology of face recognition. American Psychologist 1980;35:176-86.

[7] Benton AL. Facial recognition. Cortex 1990;26:491-9.

[8] Benton AL, Sivan AB, Hamsher K, Varney NR, Spreen O. Contribution to neuropsychological assessment. NY: Oxford University Press; 1983.

[9] Benton AL, Van Allen MW. Prosopagnosia and facial discrimination. Journal of Neurological Sciences 1972;15:167-72.

[10] Bernstein L, Beig S, Siegenthaler AL, Grady CL. The effect of encoding strategy on the neural correlates of memory for faces. Neuropsychologia 2002;40:86-98.

[11] Bruce V, Young AW. Understanding face recognition. British Journal of Psychology 1986;77:305-27.

[12] Bruyer R, Laterre C, Seron X, Feyereisen P, Strypstein E, Pierrard E, et al. A case of prosopagnosia with some preserved covert recognition of familiar faces. Brain and Cognition 1983;2:257-84.

[13] Buxbaum LJ, Glosser G, Coslett HB. Impaired face and word recognition without object agnosia. Neuropsychologia 1999;37:4150.

[14] Cabeza RK. Features are also important: contributions of featural and configural processing to face recognition. Psychological Science 2000;11:429-33.

[15] Carlesimo GA, Caltragirone C. Components in the visual processing of known and unknown faces. Journal of Clinical and Experimental Neuropsychology 1995;17:691-705.

[16] Clarke S, Lindemann A, Maeder P, Borruat F-X, Assal G. Face recognition and postero-inferior hemispheric lesions. Neuropsychologia 1997;35:1555-63.

[17] De Renzi E, Faglioni P, Grossi D, Nichelli P. Apperceptive and associative forms of prosopagnosia. Cortex 1991;27:213-21.

[18] De Renzi E, Pellegrino G. Prosopagnosia and alexia without object agnosia. Cortex 1998;34:403-15.

[19] Della Sala S, Muggia S, Spinnler H, Zuffi M. Cognitive modeling of face processing: evidence from Alzheimer patients. Neuropsychologia 1995;33:675-87.

[20] Duchaine BC. Developmental prosopagnosia with normal configural processing. NeuroReport 2000;11:79-83.

[21] Ellis HD, Shepherd JW, Davies GM. Identifications of familiar and unfamiliar faces from internal and external features: some implications for theories of face recognition. Perception 1979;8:431-9.

[22] Endo M, Takahashi K, Maruyama K. Effects of observer's attitude on the familiarity of faces: using the difference in cue value between central and peripheral facial elements as an index of familiarity. Tohoku Psychologica Folia 1984;43:23-34.
[23] Evans J, Heggs AJ, Antoun N, Hodges JR. Progressive prosopagnosia associated with selective right temporal lobe atrophy: a new syndrome? Brain 1995;118:1-13.

[24] Farah MJ. Is face recognition 'special'? Evidence from neuropsychology. Behavioural Brain Research 1996;76:181-9.

[25] Farah MJ, Wilson KD, Drain HM, Tanaka JR. What is "special" about face perception? Psychological Review 1998;105:482-98.

[26] Freire A, Lee K, Symons L. The face-inversion effect as a deficit in the encoding of configural information: direct evidence. Perception 2000;29:159-70.

[27] Gauthier I, Behrmann M, Tarr MJ. Can face recognition really be dissociated from object recognition? Journal of Cognitive Neuroscience 1999;11:349-70.

[28] Gentileschi V, Sperber S, Spinnler H. Progressive defective recognition of familiar people. Neurocase 1999;5:407-24.

[29] Hay DC, Young AW. The human face. In: Ellis AW, editor. Normality and pathology in cognitive function. London: Academic Press; 1982. p. $173-202$.

[30] Henke K, Schweinberger SR, Grigo A, Klos T, Sommer W. Specificity of face recognition: recognition of exemplars of non-face objects in prosopagnosia. Cortex 1998;34:289-96.

[31] Kanwisher N. Domain-specificity in face recognition. Nature Neuroscience 2000;3:759-63.

[32] Kracke I. Developmental prosopagnosia in Asperger syndrome: presentation and discussion of an individual case. Developmental Medicine and Child Neurology 1994;36:873-86.

[33] Lhermitte F, Pillon B. La prosopagnosie. Role de l'hemisphere droit dans la perception visuelle. Revue Neurologique 1975;131:791-812.

[34] Mack A, Rock I. Inattentional blindness. Cambridge (MA): MIT Press; 1998.

[35] Malone DR, Morris HH, Kay MC, Levin SH. Prosopagnosia: a double dissociation between the recognition of familiar and unfamiliar faces. Journal of Neurology, Neurosurgery and Psychiatry 1982;45:820-2.

[36] Mattson AJ, Levin HS, Grafman J. A case of closed prosopagnosia following moderate closed head injury with left hemisphere focal lesion. Cortex 2000;36:125-37.

[37] McKone E, Martini P, Nakayama K. Categorical perception of face identity in noise isolates configural processing. Journal of Experimental Psychology: Human Perception and Performance 2001;27:573-99.

[38] McNeil JE, Warrington EK. Prosopagnosia: a reclassification. Quarterly Journal of Experimental Psychology 1991;43A:267-87.

[39] Moscovitch M, Moscovitch D. Super face-inversion effects for isolated internal and external features, and for fractured faces. Cognitive Neuropsychology 2000;17:201-19.

[40] Moscovitch M, Winocur G, Behrmann M. What is special about face recognition? Nineteen experiments on a person with visual object agnosia, and dyslexia but normal face recognition. Journal of Cognitive Neuroscience 1997;9:555-604.

[41] Newcombe F. The processing of visual information in prosopagnosia and acquired dyslexia: functional versus physiological interpretation. In: Osborne DJ, Gruneberg MM, Eiser JR, editors. Research in psychology and medicine. London: Academic Press; 1979. p. 315-22.

[42] Nunn JA, Postma P, Pearson R. Developmental prosopagnosia: should it be taken at face value? Neurocase 2001;7:15-27.

[43] Parry FM, Young AW, Saul JSM, Moss A. Dissociable face processing impairments after brain injury. Journal of Clinical and Experimental Neuropsychology 1991;13:545-58.

[44] Rakover S, Teucher B. Facial inversion effects: parts and whole relationship. Perception and Psychophysics 1997;59:752-61.

[45] Ross ED. Sensory-specific and fractional disorders of recent memory in man. I. Isolated loss of visual recent memory. Archives of Neurology 1980;37:193-200.

[46] Shuttleworth EC, Syring V, Allen N. Further observations on the nature of prosopagnosia. Brain and Cognition 1982;1:307-22.

[47] Takahashi N, Kawamura M, Hirayama K, Shiota J-L, Isono O. Prosopagnosia: a clinical and anatomical study of four patients. Cortex 1995;31:317-29. 
[48] Tyrrell PJ, Warrington EK, Frackowiak RS, Rossor MN. Progressive degeneration of the right temporal lobe studied with positron emission tomography. Journal of Neurology, Neurosurgery and Psychiatry 1990;53:1046-50.

[49] Vuilleumier P. Faces call for attention: evidence from patients with visual extinction. Neuropsychologia 2000;38:693-700.

[50] Vuilleumier P, Sagiv N. Two eyes make a pair: facial organization and perceptual learning reduce visual extinction. Neuropsychologia 2001;39:1144-9.

[51] Warrington EK. Recognition memory test. Windsor (UK): NFERNELSON; 1984.
[52] Warrington EK, Ackroyd C. The effect of orienting tasks on recognition memory. Memory and Cognition 1975;3:140-2.

[53] Young AW. Face recognition. In: d'Ydewalle G, Eelen P, Bertelson $\mathrm{P}$, editors. International perspectives on psychological science, vol. 2. Hillsdale (NJ): LEA; 1994. p. 1-27.

[54] Young AW, Hay DC, McWeeny KH, Flude BM. Matching familiar and unfamiliar faces on internal and external features. Perception 1985;14:737-46.

[55] Young AW, Newcombe F, de Haan E, Small M, Hay DC. Face perception after brain injury: selective impairments affecting identity and expression. Brain 1993;116:941-59. 